\title{
Development And Validation of A Pyroptosis - Related Signature And Immune Microenvironment Infiltration In Cutaneous Melanoma
}

\section{Wei Dalong}

Affiliated Hospital of Youjiang Medical University for Nationalities

\section{Xiaoling Lan}

AffiliYoujiang Medical University for Nationalities

Qiang Tang

Affiliated Hospital of Youjiang Medical University for Nationalities

\section{Zhiqun Huang}

Affiliated Hospital of Youjiang Medical University for Nationalities

Qianli Tang ( $\nabla$ htmgx@ymcn.edu.cn )

Youjiang Medical University for Nationalities Baise https://orcid.org/0000-0001-5241-6372

\section{Primary research}

Keywords: Cutaneous melanoma, Pyroptosis, Immune Microenvironment

Posted Date: August 26th, 2021

DOl: https://doi.org/10.21203/rs.3.rs-772494/v1

License: (c) (i) This work is licensed under a Creative Commons Attribution 4.0 International License. Read Full License 


\section{Abstract}

Background: Cutaneous melanoma is cancer that is both malignant and aggressive, with a poor prognosis. Pyroptosis can affect the prognosis of cancer patients by controlling tumor cell growth, migration, and metastasis, as well as is closely related to the tumor immune microenvironment. The significance of pyroptosis-related genes (PRGs) in cutaneous melanoma, however, is unknown.

Methods: The training set and external validation sets were cutaneous melanoma samples from The Cancer Genome Atlas (TCGA) database and Gene Expression Omnibus (GEO), respectively. By using univariate Cox regression analysis and selection operator (Lasso) regression model, prognostic genes for overall survival (OS) were found. Candidate genes that were screened were used to calculate risk scores and construct a PRG risk model. The Kaplan Meier curve, time-dependent receiver operating characteristic (ROC) curve, and area under the curve (AUC) were used to assess the functional and prognostic usefulness of gene signatures in the risk model. Furthermore, to speculate on the activity of immune cell infiltration and immune-related pathways in the tumor immune microenvironment and calculate corresponding scores, a single sample gene set enrichment analysis (ssGSEA) was used.

Results: An eight PRGs risk signature (AIM2, CASP4, CASP5, CASP8, IL18, NLRC4, NLRP6, PRKACA) were conducted and divided all cutaneous melanoma patients in the TCGA cohort into two groups: Low-risk and High-risk. Both the training and external validation sets showed that patients in the low-risk group showed a significantly higher likelihood of survival than those in the high-risk group $(p<0.001)$. Except for PRKACA, all the other eight PRGs in our study appeared to be longer survival times for patients. The results of SSGSEA in terms of 16 types of immune cells and the activity of 13 immune-related pathways showed that the High-risk group had lower immune pathway activity and lower levels of immune cell infiltration. In conclusion, the PRG-signature may be a significant predictor of prognosis and may play an essential role in UM patients' tumor immunity.

\section{Introduction}

Melanoma of the skin is cancer that is both malignant and aggressive that is the most lethal form of skin cancer, accounting for more than $80 \%$ of all skin cancer deaths $[1,2]$, with rapidly rising incidence over the past decades. Invasive melanoma will be diagnosed in roughly 76,100 new cases in the United States, with 9710 fatalities expected[3]. Because of enormous efforts toward early diagnosis and treatments, following full excision and systemic therapy, early-stage cutaneous melanoma has a better clinical outcome. Despite recent improvements, melanoma's propensity for invasion, metastasis, and treatment resistance, the prognosis for this disease remains poor. Patients with advanced-stage melanoma still have a poor prognosis, with 5-year overall survival rates ranging from 45 percent for stage III to 18 percent for stage IV $[4,5]$.

A growing number of reports indicated that tumor immune microenvironment (TIME) plays a determining factor in modifiable for tumorigenesis, progression, and metastasis. Melanoma cells come into contact 
with both the innate and adaptive immune systems, causing immune cells with tumor monitoring and killing functions to become unresponsive, tumor-protective immune cells to become active, and TIME to form with abnormal components and functions, all of which contribute to immune escape and early melanoma metastasis[6, 7]. Patients with a high amount of tumor immune infiltration have a greater chance of responding to immunotherapies and having better clinical outcomes [2, 8]. Clarifying the complex mechanisms of tumor immune regulation and the prognostic or predictive biomarkers associated with TIME may be useful in identifying molecular targets and guiding patient treatment, as well as an effective way to find new therapies for advanced, recurrent, and metastatic melanoma.

Pyroptosis is a specific category of programmed cell necrosis that results in inflammation disintegration in cells[8]. Through cell swelling, lysis, and the release of many pro-inflammatory chemicals, pyroptosis regulates programmed cell necrosis. Several risk-related signaling molecules and cytokines are activated and disseminated after pyroptosis, resulting in a significant inflammatory cellular response and immune system activation[9]. Pyroptosis has a significant pro-inflammatory effect that is linked to the control of the tumor immune microenvironment, which is crucial for tumor growth[10]. Moreover, despite the fact that pyroptosis has been linked to cancer in numerous studies, the relationship between pyroptosis and anticancer immunity is still a mystery [9].

Considering the available findings, we understand that pyroptosis and TIME play a dual and critical function in the growth of tumors as well as in the anti-tumor process. However, its specific functions in cutaneous melanoma have not been well investigated. As a result, the fast identification of novel biomarkers and oncogenic agents in melanoma is important.

To the best of our knowledge, there are limited studies that focus on the relationship between TIME, pyroptosis, and cutaneous melanoma prognosis. Accordingly, we conducted a comprehensive investigation to develop a prognosis model for pyroptosis-related genes (PRGs) and identify distinct risk score groups, as well as to investigate the relationship between pyroptosis and TIME and to assess the prognostic relevance of these genes.

\section{Materials And Methods}

\section{Sources of Cutaneous Melanoma Patient Datasets}

The Cancer Genome Atlas (TCGA) data portal was used to obtain RNA sequencing (RNA-seq) data from 470 people with cutaneous melanoma, as well as their clinical characteristics (http://www.cbioportal.org/ repository). The mRNA expression patterns of 214 melanoma samples were collected from the GSE65904 mRNA microarray (GPL10558 Illumina HumanHT-12 V4.0) from the GEO database (https://www.ncbi.nlm.nih.gov/geo/). The TCGA and GEO data portals were used to collect clinical details about the patients, such as survival time and status.

\section{Data preprocessing}


The previous review contributed 33 PRGs to our research[10-12]. Protein-Protein Interaction Networks (PPI) of 33 PGRs were presented in Fig.1. Because the data were extracted from public databases following the relevant strictly approved publication guidelines, ethics committee approval was not required.

When pre-processing data, if duplicate or incompletely documented cases were found, all records were removed from further analysis. After removing incomplete information records, a total of 454 cases of TCGA cohort and 214 cases of GEO data portal were available for further analysis.

\section{Construction of the PRG signature and Risk Score Prognostic Analysis}

To investigate the role of PRGs in prognosis prediction. We further performed univariate Cox regression analysis to the 33 PRGs in combination with the survival information in the TCGA cohorts as the training cohort. 15 overall survivals (OS) related PRGs were subsequently screened for further construct the prognostic signature. These OS-related PRGs were utilized to further create the prognosis model by Lasso Cox regression model to reduce the number of candidate genes and build a predictive signature $(R$ package "glmnet", "survival"). Ultimately, after centralizing and standardizing the linked expression value and coefficients of the nine PRGs, risk scores were calculated based on the related expression value and coefficients of the nine PRGs. The formula for calculating the risk score was as obeys: , (X: the coefficients of the nine-PRGs, Y: level of gene expression in the nine-PRGs,: each of the nine-PRGs). According to the risk score formula above, each patient of the TCGA cohort was calculated the risk value. The median risk score in each cohort was used to classify cutaneous melanoma patients into low- and high-risk categories.

\section{Immune cells infiltration and immune score calculation}

We measured and calculated scores for the level of tumor immune infiltration in patients with cutaneous melanoma in the TCGA and GEO cohorts using the ssGSEA in the "GSVA" R package.

\section{Statistical methods}

Different risk subgroups or expression levels of PRGs of cutaneous melanoma patients were applied by two-sided log-rank test and survival curves were visualized by R package 'survival'. Receiver operating characteristic (ROC) curves were produced with the R package 'timeROC' to assess the sensitivity and specificity of the PRG-signature model[13]. The area under the curve (AUC) was then calculated as the main quantitative parameter[14]. Univariate Cox regression was a critical analytical approach for determining the PRG-independent signature's predictive function based on clinical and survival data. Pearson correlation analysis (PCA) was applied to calculate the correlation between risk scores. The statistical analysis methods mentioned above were based on an implementation of the R programming language (version 4.1.0, https://www.r-project.org/). All statistical tests were two-sided, with statistical significance defined as $\mathrm{P}<0.05$. 


\section{Results}

\section{Development of the PRGs-signature in cutaneous melanoma patients}

To investigate the role of PRGs in prognosis signature. Then, univariate Cox regression analysis was performed, and finally 15 PRGs associated with OS were screened $(p<0.05)$, comprising 12 protective PRGs $(H R<1)$ and three dangerous PRGs $(H R>1)$. A forest plot revealed significant PRGs (Fig. 2A). The Lasso Cox regression model was subsequently applied to 15 PRGs relate to OS to establish a prognostic model for cutaneous melanoma patients in the TCGA cohort. Optimum $\lambda$ values were used to identify 9PRGs signatures (Fig. 2 B and C). In Fig.2 D, the coefficients of the nine ARGs were displayed from low to high. To ascertain the predictive value of PRGs-signature in terms of prognosis of cutaneous melanoma patients. The 454 cutaneous melanoma samples of TCGA cohort were equally divided into 2 groups of Low-risk combined with High-risk (Fig. 2E). According to the findings of principal component analysis (PCA), patients in terms of their different risk subgroups were well divided into two clusters (Fig. 2F). Similarly, the results of t-distributed stochastic neighbor embedding (t-SNE) show distinct clustering patterns. (Fig. 1G). According to the result of t-SNE and PCA, patients of TCGA with different risk strata had varied gene expression and pyroptosis status.

Patients with high-risk scores had more mortality and shorter survivorship (Fig.2H). Survival analysis in visualization by Kaplan-Meier OS curves was indicated that the low-risk cutaneous melanoma subgroup had higher rates of survival and longer survival duration of time compared to the high-risk subgroup $(P<0.001)$ (Fig. 2l). A time-dependent ROC analysis was used to measure the sensitivity and specificity of the prognostic model. For 1,2 , and 3 years, the area under the ROC curve (AUC) was $0.682,0.698$, and 0.659 , respectively. Which implied that the predictive capability of the model is good (Fig.2J).

\section{External validation of the risk signature}

The validation set consisted of 214 cutaneous melanoma patients from a Gene Expression Omnibus (GEO) cohort (GSE65904). The "Scale" function was used to standardize the gene expression data before further investigation. The median risk score for the GEO cohort was calculated according to the risk score formula for the TCGA cohort, and then the patients were divided equally into two groups: a low-risk group and a high-risk group. Higher risk scores were associated with a lower OS rate and a shorter OS duration, according to the results of survival analysis. (Fig.3A). The distributions of OS time and status across patients also supported this conclusion (Fig. 3B -C). The risk categorization approach employed was able to separate the patients into two clusters more effectively (Fig. 3D). ROC curves indicated that the risk score preserved a high level of prognostic prediction accuracy in the GEO cohort (Fig. 3E).

\section{The prognostic value of the PRGs}

To compare survival difference low- and high-expression of 9-PRGs in the TCGA cohort. The connection between OS and low- and high expression levels of PRGs were discovered using survival analysis. Differential expression of 8-PRGs was later discovered to be linked to melanoma OS. cutaneous 
melanoma patients with high levels of AIM2, CASP4, CASP5, CASP8, IL18, NLRC4, NLRP6 expression had improved clinical outcomes, suggesting that these proteins might be protective PRGs (Fig.4 A-G).

Nevertheless, Patients with high PRKACA expression levels had poorer clinical outcomes, suggesting that PRKACA may act as hazardous PRGs in cutaneous melanoma (Fig. $4 \mathrm{H}$ ).

\section{TIME infiltration differences between two PRG-related risk subgroups}

In the TCGA cohort, we also utilized SSGSEA to investigate the relationship between PRG features and the tumor immune microenvironment in low- and high-risk cutaneous melanoma subgroups (Fig. $5 \mathrm{~A}$ and B). Low-risk groups had higher 16 kinds of immune cells infiltration and 13 immune-related pathways. The GEO cohort had similar results (Fig. $5 \mathrm{C}$ and D). When comparing the high-risk and low-risk groups, the immunological microenvironment was changed in the high-risk group, as demonstrated by reduced immune cell infiltration and fewer immune-related pathways.

\section{Discussion}

Along with the booming evolution of biotechnological and bioinformatics science, genomic analysis had been widely applied to search cancer biomarkers or develop diagnostic and prognostic models $[15,16]$. However, only a few related research focused on the creation of prognostic prediction models for cutaneous melanoma patients. With the help of the Bioconductor in R project, we combined univariate Cox regression and Lasso Cox regression models to screen for PRGs associated with OS, and further developed a prognostic PRG-signature to distinguish patients with different clinical outcomes in the present study. Our study generated an 8 PRGs risk signature (AIM2, CASP4, CASP5, CASP8, IL18, NLRC4, NLRP6, PRKACA) and demonstrated that these regulators OS in patients with cutaneous melanoma. In our present investigation, CASP4, CASP5, and CASP8 appear to be oncogenes that prolong the survival and improve the clinical prognosis of cutaneous melanoma patients maybe through inflammatory and apoptotic pathways respectively. Except for PRKACA, all the other eight PRGs in our study appeared to be suppressants of cancers, which resulted in longer survival times for patients. We created a risk score based on PRG features and discovered that high-risk patients had a worse clinical prognosis and a shorter survival time. The high-risk group had considerably lower scores for immune cell infiltration and immunological pathways than the low-risk group.

Pyroptosis is a type of programmed cell necrosis that has just recently been identified, however, it plays a crucial and dual role in the processes that promote and prevent carcinogenesis. For one thing, pyroptosis can prevent tumor formation and progression; for another, as a form of proinflammatory death, pyroptosis can provide a favorable microenvironment for tumor cell proliferation, promoting tumor growth $[12,17]$. The mechanism of the trigger of pyroptosis is through the activation of the inflammasome, which is a multimolecular complex containing pattern-recognition receptors (PRR) stimulated by an inflammatory response. PRR recognizes certain pathogen-associated molecular patterns (PAMPs) induced by invading pathogens and certain damaged-associated molecular patterns (DAMPs) derived from endogenous pathogens[18]. 
Many previous studies have shown that AIM2-like receptors (ALRs), Toll-like receptors (TLRs), intracellular nucleotide-binding oligomerization domain (NOD)-like receptors (NLRs) are all associated with pyroptosis [19]. NLRs include eight members (NLRP1, NLRP2, NLRP3, NLRP6, NLRP7, NLRP12, NLRC4, and NAIP) and AIM2 can activate inflammasomes. Assembly of the inflammasome initiates activation of downstream Caspase 1 is a self-activating proenzyme that converts to a protease and has a physiological role. Caspases- 1 activation causes the processing and maturation of pro-inflammatory cytokines such as interleukin (IL)-1 and IL-18, as well as pyroptosis [20].

Pyroptosis has a function in a variety of malignancies. Pyroptosis is also associated with gastric cancer[21], breast cancer[22], Cervical cancer[23]. Given the preceding, growing data suggests that pyroptosis and associated genes have a dual anticancer and tumor-promoting role in tumorigenesis. Assessing the predictive significance of cutaneous melanoma using only a few pyroptosis regular expression patterns is currently insufficient. Therefore, we investigated immune cells directly linked to pyroptosis, as well as a predictive signature based on the impact of the implicated pathways on the TIME. The development of pyroptosis in human disease, as well as its mechanisms, has been extensively researched. Like apoptosis and autophagy, pyroptosis will have a huge influence on cancer detection and therapy. Pyroptosis is increasingly being investigated as an anti-tumor treatment, and our findings show that pyroptosis coupled with immunotherapy to enhance patient prognosis might be a promising therapeutic approach.

Melanoma is one of the malignancies with the highest immunogenicity and has the highest potential to activate immunological responses due to its high genomic mutational load. The TIME is also important for tumor outcome and prognosis. Innate and adaptive immunity act together to prevent melanoma in its early stages and to offer long-term protection against recurrence. The fast and non-specific anti-tumor responses of innate immunity are critical not just for avoiding and reducing melanoma in its early stages, but also for priming strong adaptive immunity in order to provide long-term tumor-specific immune surveillance[24].

In the preliminary stages of tumour development, immune cells ,including Natural killer cells[24], Macrophages[24], neutrophils ${ }^{[25]}$, dendritic cells[26], Th (helper) cells[27], Teff/Tc (effector/cytotoxic) cells, Treg ( regulatory) cells) fulfill their proper function they produce anti-cancer actions via inducing apoptosis in altered cells, generating anti-tumor cytokines, or triggering cytotoxic responses.

By secreting cytokines, activated NK cells help to recruit antigen-presenting cells (APCs), whereas neutrophils, macrophages, and dendritic cells in the tumor niche phagocytose the dead melanoma cells. Subsequently, T cells, including T effector (Teff) cells, cytotoxic T lymphocytes (Tc), and T helper (Th) cells, are activated by APC through the presentation of cancer antigens. Long-term melanoma remission necessitates the activation and growth of these cell types[28]. However,Immune escape is a phenomena caused by the plasticity of melanoma cells, in which cancer cells have a less immunogenic phenotype and the capacity to inhibit anti-tumor immune cells in the tumor microenvironment [29]. Immune escape manifests itself in the tumor microenvironment as a reduction in immune cell infiltration. Immune scores 
calculated from gene expression data were used to indicate immune features to estimate the infiltration of immune cells in tumor microenvironment[30].

The levels of important infiltrating immune cells and immune related pathways were low in the high-risk group in the TCGA cohort, suggesting a general deterioration of immunological functions, which was confirmed in the GEO cohort. Melanomas of a high-risk group with a low level of immune cell infiltration have a poor prognosis. Immunotherapy will be more effective if there is a higher infiltration of immune cells. We investigated the predictive significance of these PRGs in the preliminary stages and offered a theoretical foundation for further melanoma study.

Cancer is a complicated, diverse disease with a variety of histological characteristics and clinical manifestations. The classification of samples according to pre-determined gene expression characteristics is a well-established method[31]. Depending on the expression of PRGs, we developed a subtyping approach for cutaneous melanoma patients. These regulators were also shown to be linked to a variety of survival hazards. Our analysis eventually resulted in three main points of agreement: (1) The expression of PRGs were entirely different between the two risk score subgroups in cutaneous melanoma patients; (2) The high-risk group was a distinct subgroup with a poor prognosis. (3) The risk score related to pyroptosis was used to represent individuals with various clinical characteristics and was linked to immunotherapy. A high-risk score indicated poor clinical traits as well as a shorter predicted survival time. The risk score has considerable significance for immunotherapy, as evidenced by TIME cell infiltration data. Few studies have focused on the PRGs signature in cutaneous melanoma, and we hoped that our findings would shed light on their roles.

Several limitations should be mentioned in our study. Firstly, we simply registered in the TCGA database to construct the PRG signature, which was then verified by a single GEO database. the accuracy and availability of our biomarker needed to be validated in more datasets, even in prospective research. Moreover, conducting multiple functional studies to elucidate the functions of the 8-PRGs in melanoma was critical. Despite its shortcomings, the predictive usefulness of the PRG signature for OS in melanoma patients cannot be overlooked. Our findings will need to be validated in the future by multi-institutional and well-designed research.

In summary, our research identified a unique genetic signature for predicting the prognosis of cutaneous melanoma patients and lays the groundwork for future research into the connection between and cutaneous melanoma tumor immune microenvironment. our two independent cohorts were used to develop and validate the prognostic predictive signature in cutaneous melanoma patients. The PRG signatures both revealed strong predictors of clinical outcome. Furthermore, the scores obtained from our risk signature based on nine PRGs were independently related for predicting OS in cutaneous melanoma patients. The tumor immune microenvironment differed significantly between PRGs-related risk subgroup. patients with higher risk scores showed an abundance of immune cell infiltration and immune pathways. Finally, the PRG-signature may be a significant predictor of prognosis and may play an essential role in UM patients' tumor immunity. 


\section{Declarations}

\section{Ethics approval and consent to participate}

Public datasets include TCGA and GEO. Ethical permission has been acquired for the patients in the database. Users can get access to pertinent data for free and create papers based on it. There are no ethical problems or any conflicts of interest because our research is based on free source data.

\section{Consent for publication}

Not applicable.

\section{Data Availability}

The TCGA (http://cancergenome.nih.gov/) and GEO (https://www.ncbi.nlm.nih. gov/geo/) websites provide access to the data used in this work.

\section{Conflict of interest}

The authors declare no competing interests.

Funding: Guangxi Medical High-level Leading Talents Training "139" Project, Special Funding for Guangxi Special Experts (No: GWKJ [2018]22\#)

\section{Author Contribution}

Qianli Tang constructed the present study. Dalong Wei and performed the data analysis, figures plotted, and writing. Data collection and critical reading of the text were handled by Xiaoling Lan and Qiang Tang. The final paper has been reviewed and approved by all writers.

\section{Acknowledgments}

The contributions of the TCGA project and the GEO repository are gratefully acknowledged by all authors.

\section{References}

1. Erdei E, Torres SM. A new understanding in the epidemiology of melanoma. Expert Rev Anticancer Ther. 2010;10:1811-23.

2. Hu B, Wei Q, Zhou C, Ju M, Wang L, Chen L, et al. Analysis of immune subtypes based on immunogenomic profiling identifies prognostic signature for cutaneous melanoma. Int Immunopharmacol. 2020;89:107162.

3. Saranga-Perry V, Ambe C, Zager JS, Kudchadkar RR. Recent developments in the medical and surgical treatment of melanoma: Medical and Surgical Treatment of Melanoma. CA A Cancer Journal for Clinicians. 2014;64:171-85. 
4. Hartman RI, Lin JY. Cutaneous Melanoma-A Review in Detection, Staging, and Management. Hematol Oncol Clin North Am. 2019;33:25-38.

5. Siegel RL, Miller KD, Jemal A. Cancer statistics, 2018: Cancer Statistics, 2018. CA: A Cancer Journal for Clinicians. 2018;68:7-30.

6. Binnewies M, Roberts EW, Kersten K, Chan V, Fearon DF, Merad M, et al. Understanding the tumor immune microenvironment (TIME) for effective therapy. Nat Med. 2018;24:541-50.

7. Cheng Y. Immune Microenvironment Related Competitive Endogenous RNA Network as Powerful Predictors for Melanoma Prognosis Based on WGCNA Analysis. Front Oncol. 2020;10:19.

8. Kovacs SB, Miao EA. Gasdermins. Effectors of Pyroptosis. Trends Cell Biol. 2017;27:673-84.

9. Tang R, Xu J, Zhang B, Liu J, Liang C, Hua J, et al. Ferroptosis, necroptosis, and pyroptosis in anticancer immunity. J Hematol Oncol. 2020;13:110.

10. Ye Y, Dai Q, Qi H. A novel defined pyroptosis-related gene signature for predicting the prognosis of ovarian cancer. Cell Death Discov. 2021;7:71.

11. Karki R, Kanneganti T-D. Diverging inflammasome signals in tumorigenesis and potential targeting. Nat Rev Cancer. 2019;19:197-214.

12. Xia X, Wang X, Cheng Z, Qin W, Lei L, Jiang J, et al. The role of pyroptosis in cancer: pro-cancer or pro-“host"? Cell Death Dis. 2019;10:650.

13. Blanche P, Dartigues J-F, Jacqmin-Gadda H. Estimating and comparing time-dependent areas under receiver operating characteristic curves for censored event times with competing risks. Stat Med. 2013;32:5381-97.

14. Zheng Z, Zhang L, Tu Z, Deng Y, Yin X. An autophagy-related prognostic signature associated with immune microenvironment features of uveal melanoma. Bioscience Reports. 2021;15.

15. Shao W, Yang Z, Fu Y, Zheng L, Liu F, Chai L, et al. The Pyroptosis-Related Signature Predicts Prognosis and Indicates Immune Microenvironment Infiltration in Gastric Cancer. Front Cell Dev Biol. 2021;9:676485.

16. Tu Z, Wu L, Wang P, Hu Q, Tao C, Li K, et al. N6-Methylandenosine-Related IncRNAs Are Potential Biomarkers for Predicting the Overall Survival of Lower-Grade Glioma Patients. Front Cell Dev Biol. 2020;8:642.

17. Georgescu SR, Tampa M, Mitran Cl, Mitran MI, Caruntu C, Caruntu A, et al Tumour Microenvironment in Skin Carcinogenesis. In: Birbrair A, editor. Tumor Microenvironments in Organs [Internet]. Cham: Springer International Publishing; 2020 [cited 2021 Jul 18]. p. 123-42. Available from: http://link.springer.com/10.1007/978-3-030-36214-0_10.

18. Pandey A, Shen C, Man SM. Inflammasomes in Colitis and Colorectal Cancer: Mechanism of Action and Therapies. Yale J Biol Med. 2019;92:481-98.

19. Lamkanfi M, Dixit VM. Mechanisms and functions of inflammasomes. Cell. 2014;157:1013-22.

20. Kim YK, Shin JS, Nahm MH. NOD-Like Receptors in Infection, Immunity, and Diseases. Yonsei Med J. 2016;57:5-14. 
21. Fang Y, Tian S, Pan Y, Li W, Wang Q, Tang Y, et al. Pyroptosis: A new frontier in cancer. Biomed Pharmacother. 2020;121:109595.

22. Draganov D, Gopalakrishna-Pillai S, Chen Y-R, Zuckerman N, Moeller S, Wang C, et al. Modulation of P2X4/P2X7/Pannexin-1 sensitivity to extracellular ATP via Ivermectin induces a non-apoptotic and inflammatory form of cancer cell death. Sci Rep. 2015;5:16222.

23. So D, Shin H-W, Kim J, Lee M, Myeong J, Chun Y-S, et al. Cervical cancer is addicted to SIRT1 disarming the AIM2 antiviral defense. Oncogene. 2018;37:5191-204.

24. Marzagalli M, Ebelt ND, Manuel ER. Unraveling the crosstalk between melanoma and immune cells in the tumor microenvironment. Semin Cancer Biol. 2019;59:236-50.

25. Huang Z, Gan J, Long Z, Guo G, Shi X, Wang C, et al. Targeted delivery of let-7b to reprogramme tumor-associated macrophages and tumor infiltrating dendritic cells for tumor rejection. Biomaterials. 2016;90:72-84.

26. Giavina-Bianchi MH, Giavina-Bianchi Junior PF, Festa Neto C. Melanoma: tumor microenvironment and new treatments. An Bras Dermatol. 2017;92:156-66.

27. Facciabene A, Motz GT, Coukos G. T-regulatory cells: key players in tumor immune escape and angiogenesis. Cancer Res. 2012;72:2162-71.

28. Simiczyjew A, Dratkiewicz E, Mazurkiewicz J, Ziętek M, Matkowski R, Nowak D. The Influence of Tumor Microenvironment on Immune Escape of Melanoma. IJMS. 2020;21:8359.

29. Jenkins RW, Barbie DA, Flaherty KT. Mechanisms of resistance to immune checkpoint inhibitors. Br J Cancer. 2018;118:9-16.

30. Sheng Y, Yanping C, Tong L, Ning L, Yufeng L, Geyu L. Predicting the Risk of Melanoma Metastasis Using an Immune Risk Score in the Melanoma Cohort. Front Bioeng Biotechnol. 2020;8:206.

31. Cristescu R, Lee J, Nebozhyn M, Kim K-M, Ting JC, Wong SS, et al. Molecular analysis of gastric cancer identifies subtypes associated with distinct clinical outcomes. Nat Med. 2015;21:449-56.

\section{Figures}




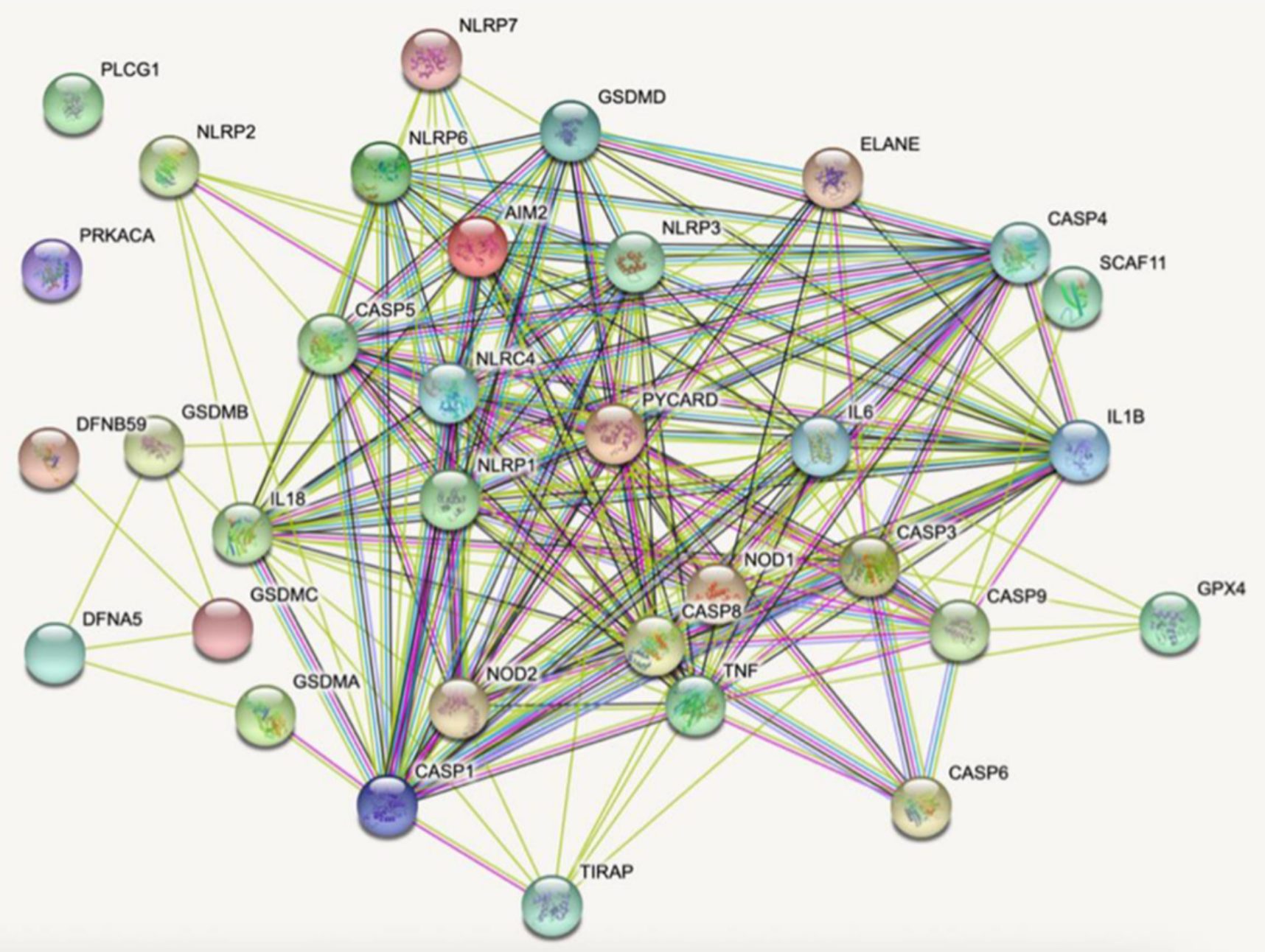

Figure 1

The PPI network downloaded from the STRING database indicated the interactions among all PRGs. 

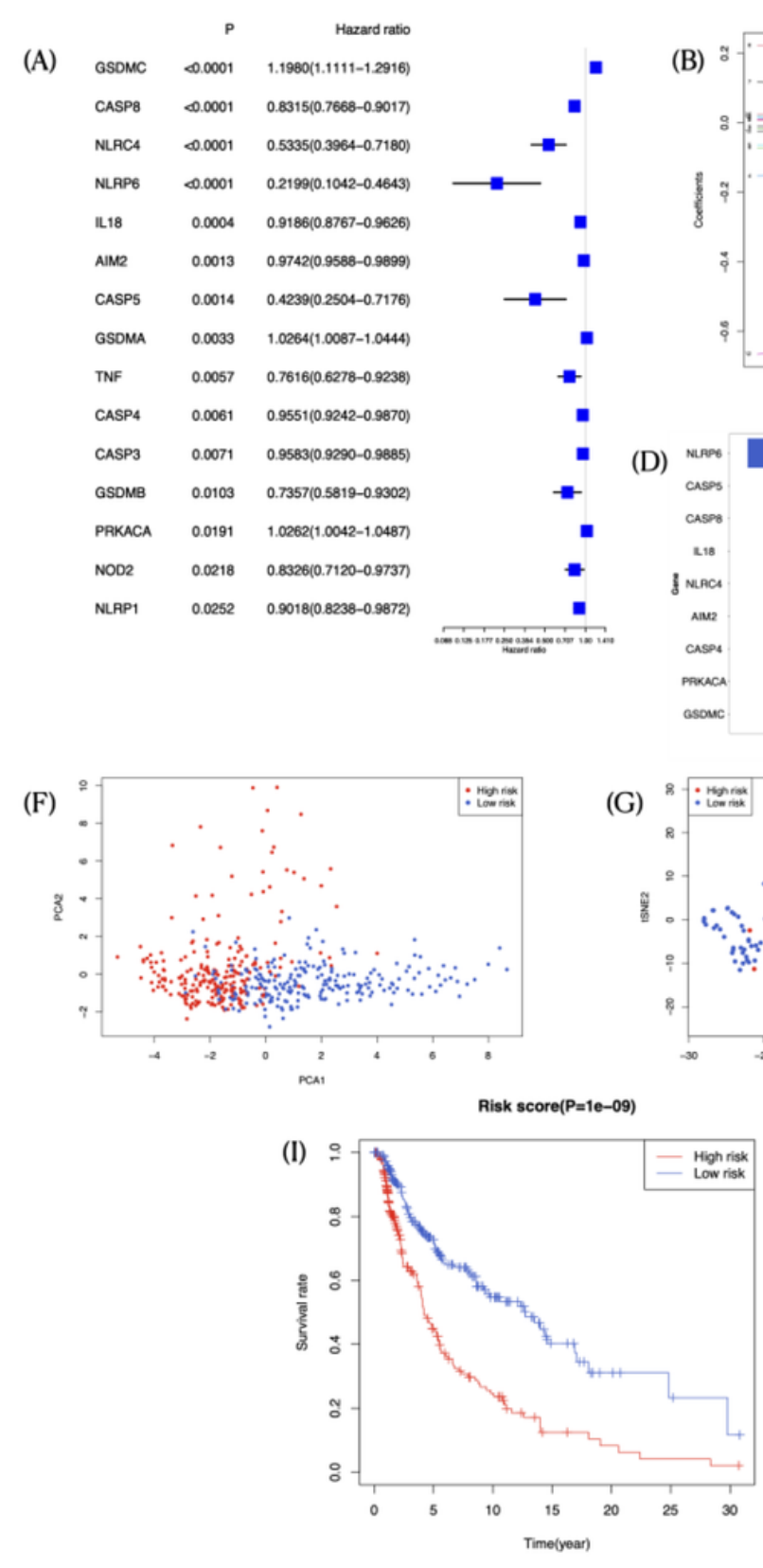

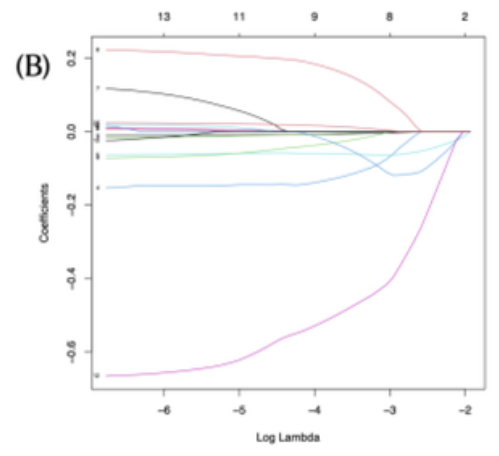

(D)

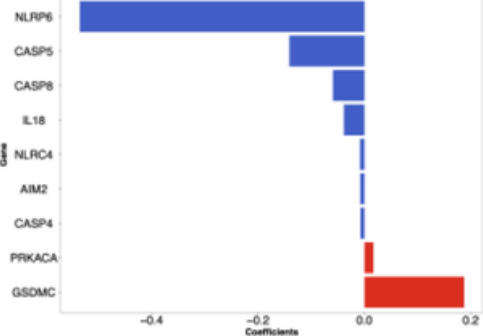

(G)

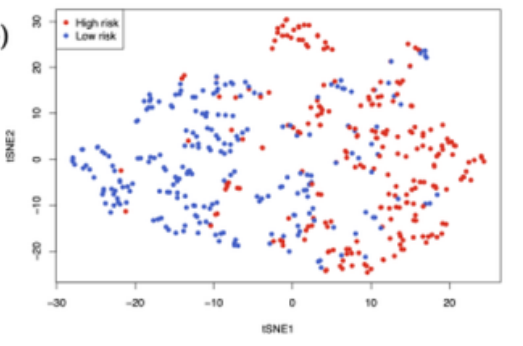

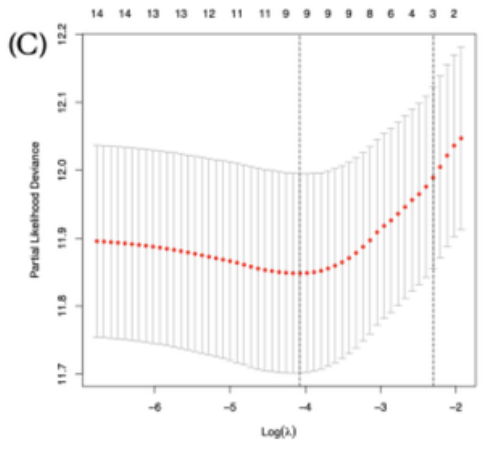

(E)

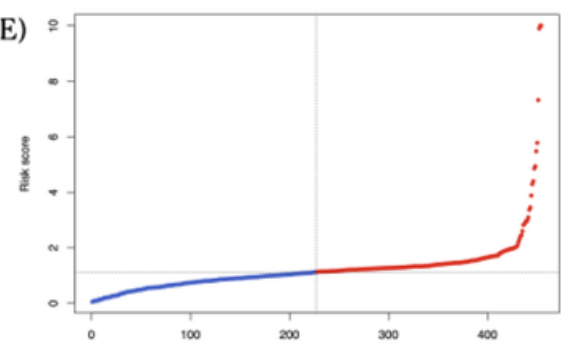

(H)

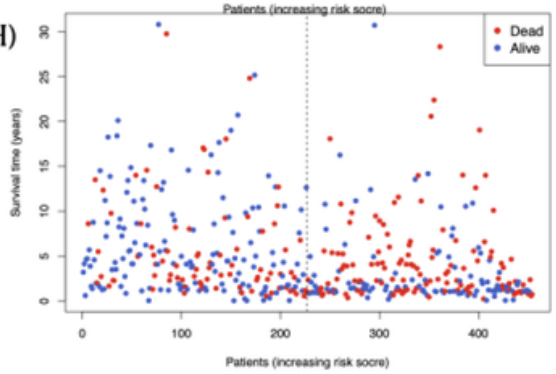

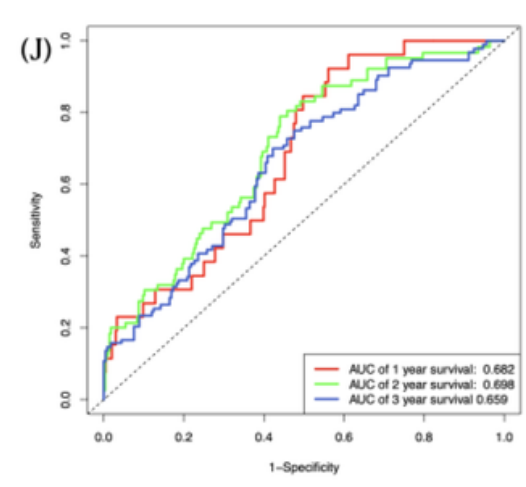

\section{Figure 2}

Construction of the PRG-signature (A) OS univariate cox regression analysis for each PRG, with a concentration on 15 PRGs linked to OS $(p<0.05)$. ( $B, C)$ The minimal criteria and coefficients for generating the PRG-signature were calculated using Lasso regression. (C) The coefficients of the nine ARGs are displayed in the histogram. (E) Patients are assigned to groups based on their risk scores. (F) PCA plot for patients in the TCGA cohort based on the risk score. (G) t-SNE plot for patients in TCGA cohort on the basis of the risk score. $(\mathrm{H})$ The distribution of survival status in terms of PRG-related risk scores for individual samples in the TCGA cohort (The low-risk populations are on the left side of the dotted line, while the high-risk ones are on the right.). (I) Kaplan-Meier curves for the OS of high-risk and low-risk patients. $(\mathrm{J})$ The prediction effectiveness of the risk score was proven using ROC curves. 


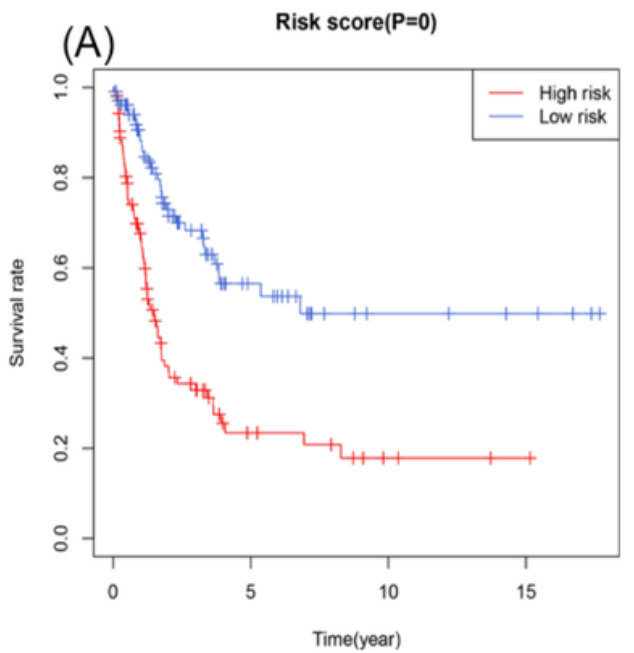

(B)

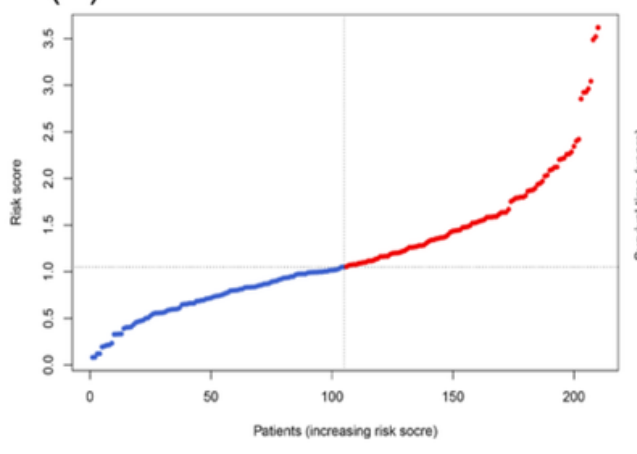

(C)

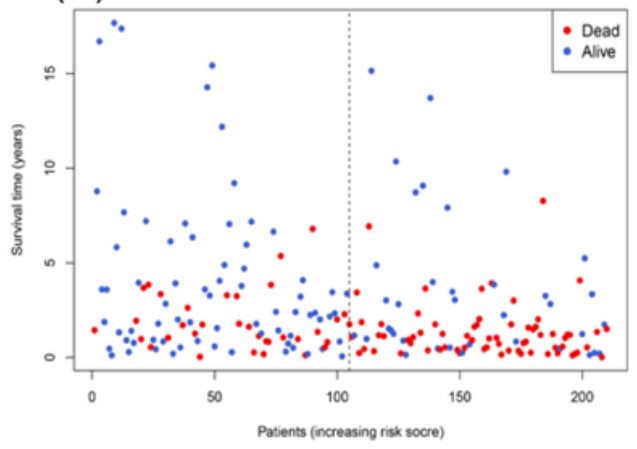

(D)

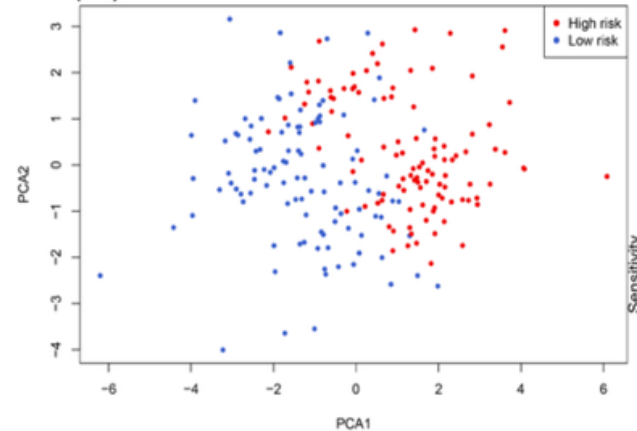

(E)

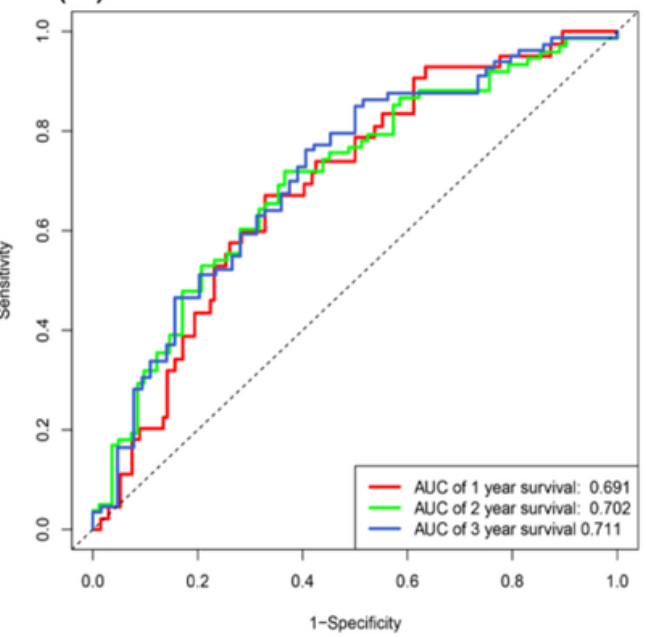

Figure 3

Validation of PRGs-related risk models in the GEO cohort. (A) The Kaplan-Meier curves are used to compare the OS of two groups. (B) Distribution of patients in the GEO cohort with PRGs-related risk scores. (C) The distribution of survival status in terms of PRG-related risk scores for individual samples in the GEO cohort (The low-risk populations are on the left side of the dotted line, while the high-risk ones are on the right.). (D)PCA plot for patients in the TCGA cohort based on the risk score. (E) Time-dependent ROC curves for patients in the GEO cohort. 
(A)

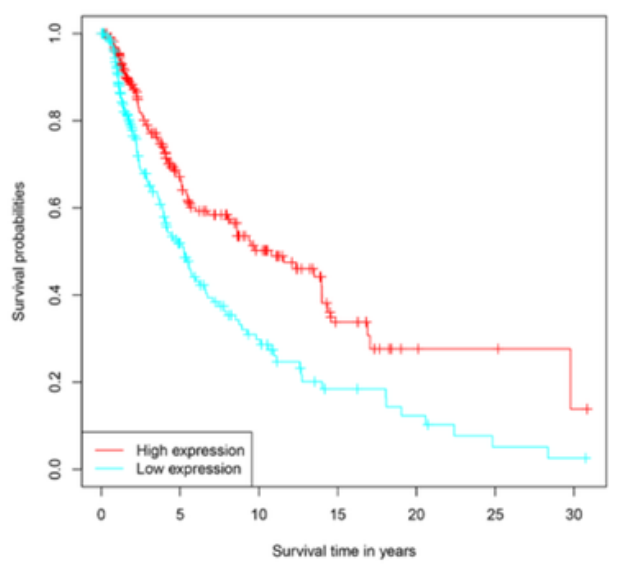

(D)

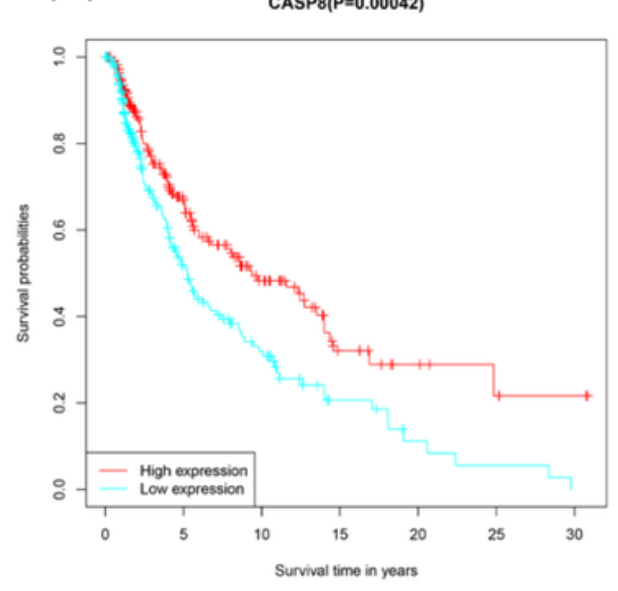

(G)

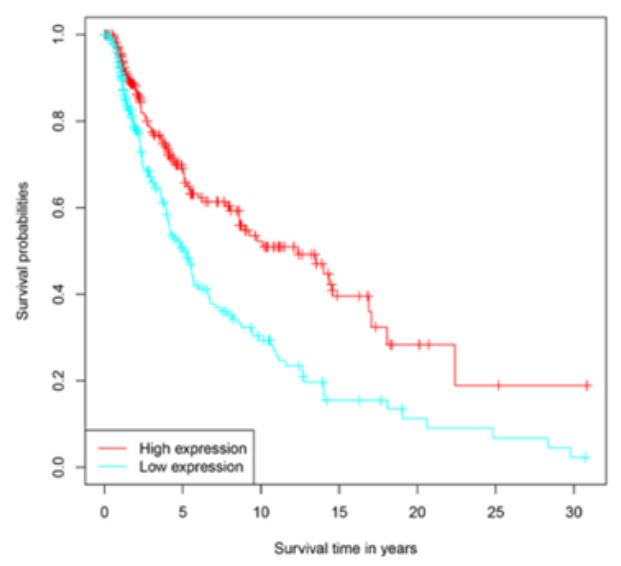

(B)

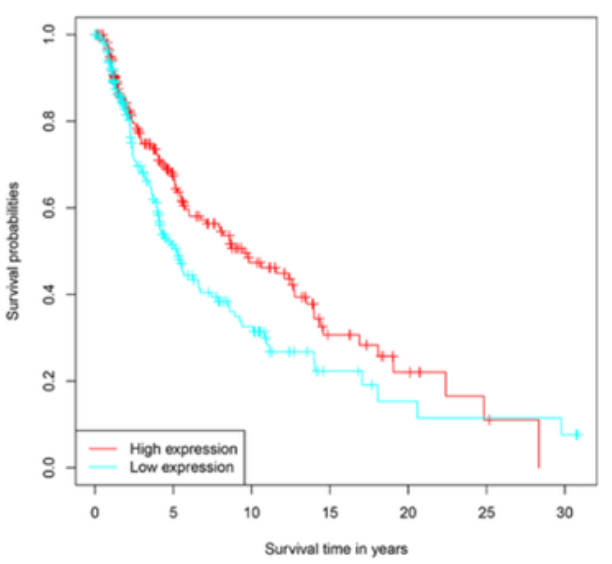

(E)

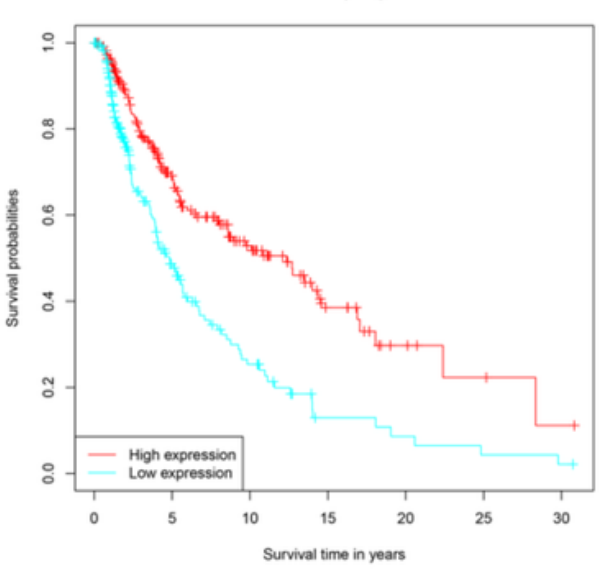

(H)

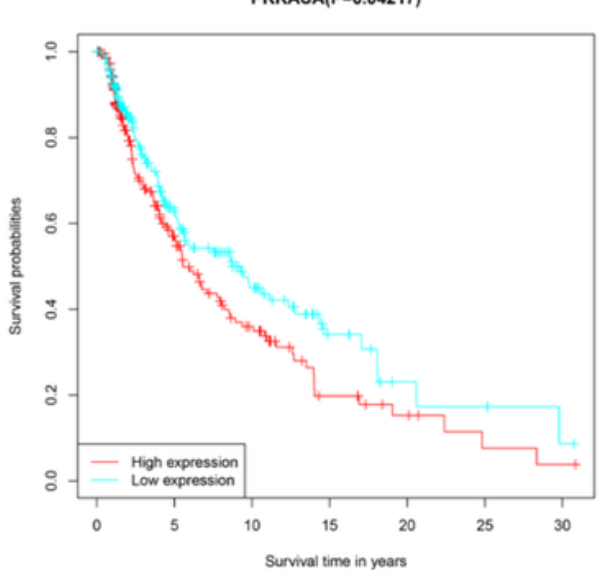

(C)

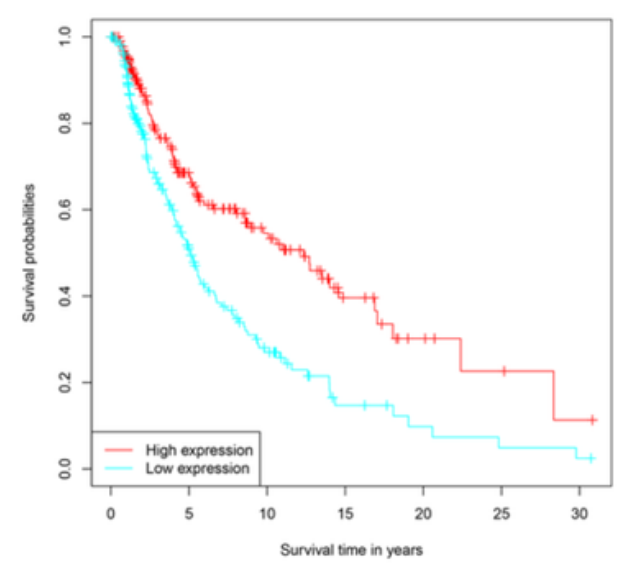

(F)

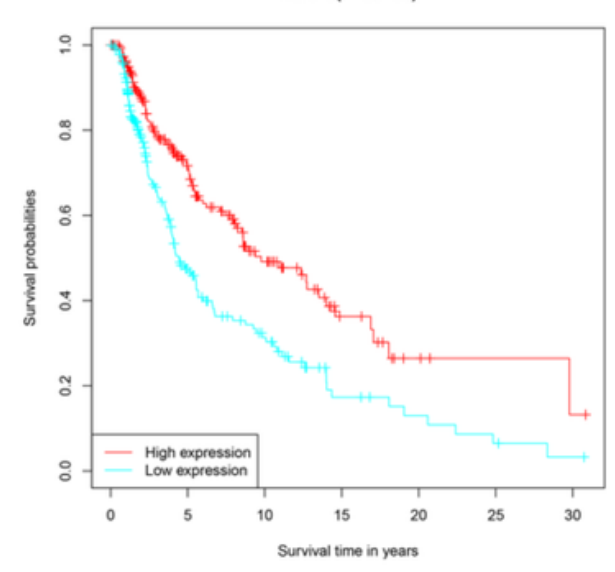

\section{Figure 4}

Prognostic roles of the nine PRGs Survival analysis represented high expressions of AIM2 (A), CASP4 (B), CASP5 (C), CASP8 (D), NLRC4 (E), NLRP6 (F), IL18(G) were correlated with better clinical outcomes of patients. Survival analysis represented high expressions of PRKACA $(H)$ was correlated with worse clinical outcomes 

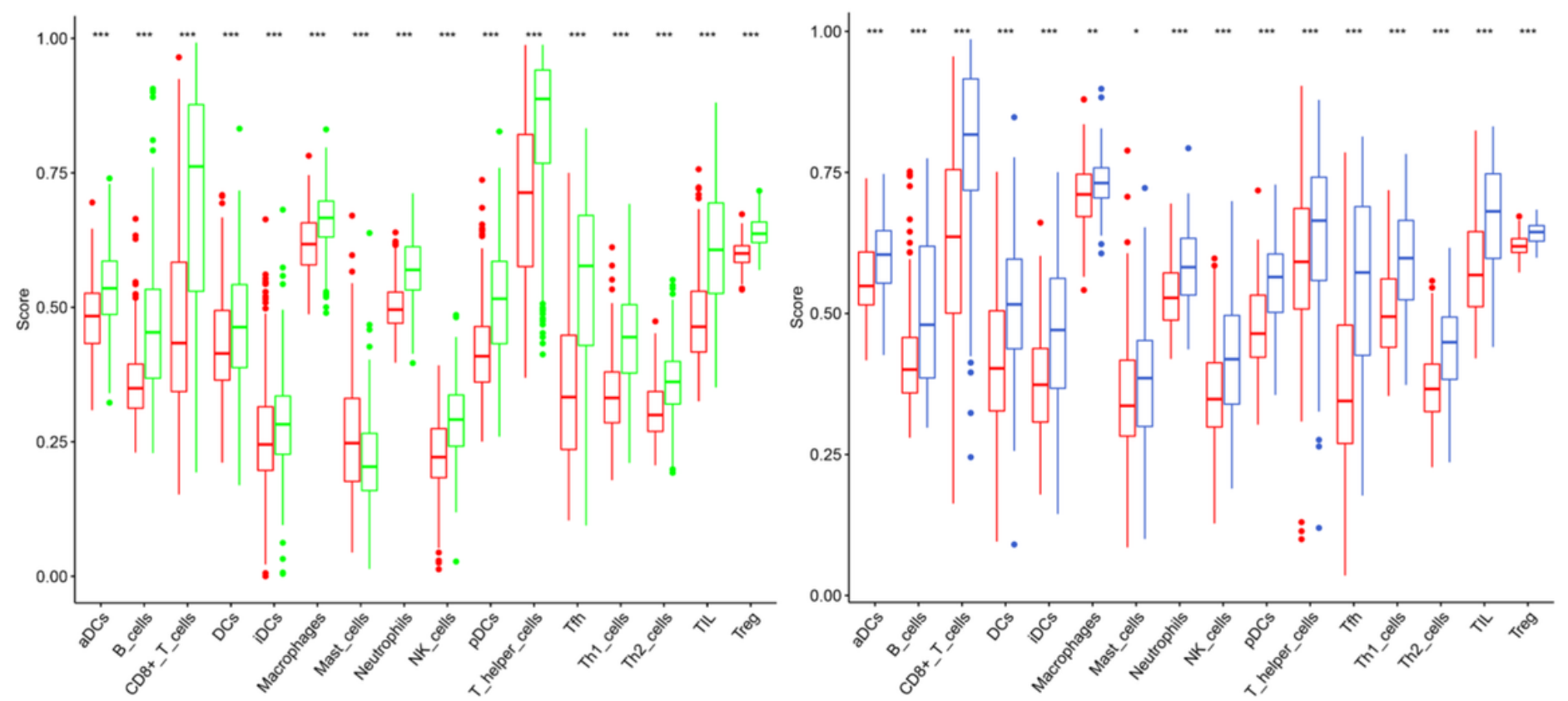

(B)

(D) Group 追 High 追 Low
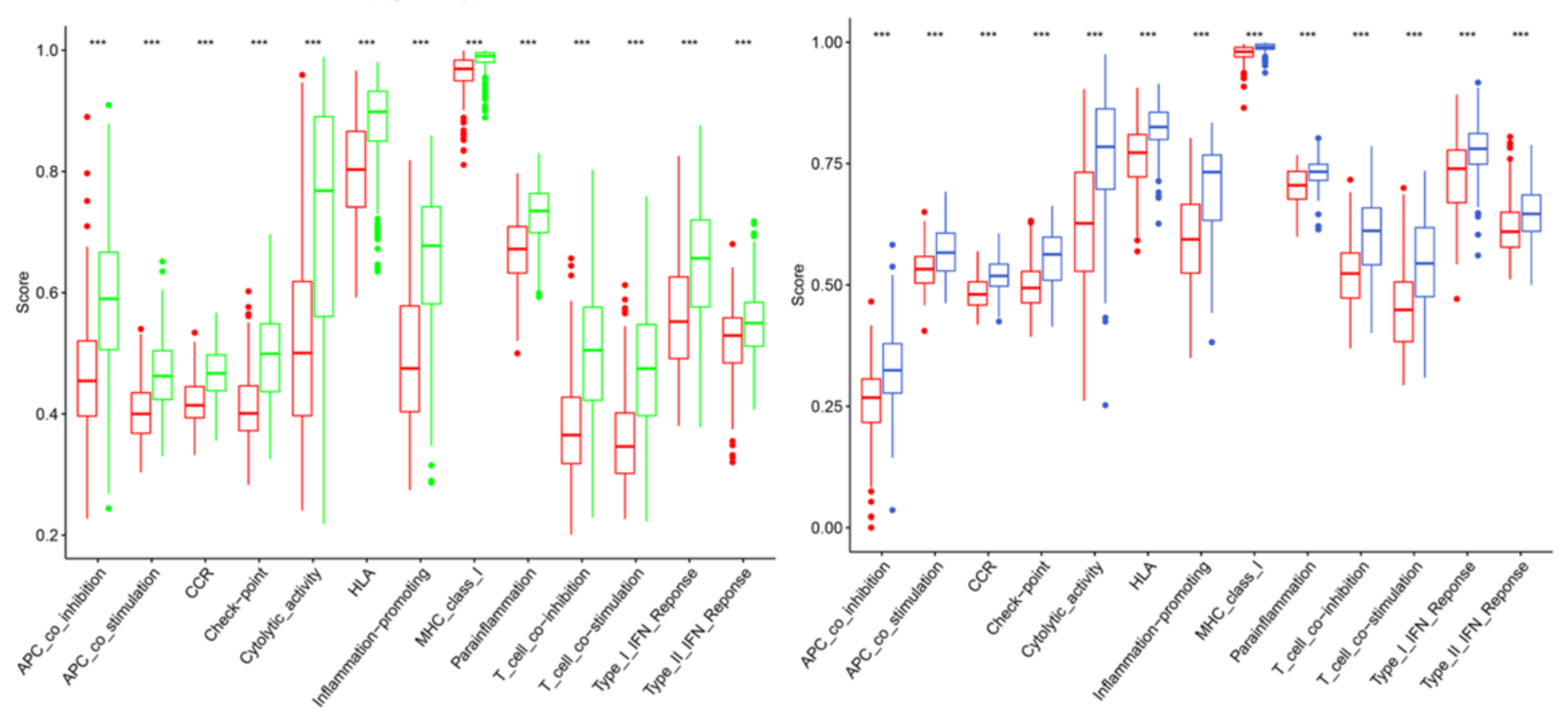

\section{Figure 5}

Comparison of the TIME infiltration in subgroups A and B In the TCGA cohort, the enrichment scores of immune cells and immune-related pathways were compared between two PRG-related risk subgroups. $C$ and D In the GEO cohort, TIME infiltration was compared between the low-risk (blue box) and high-risk (red box) groups. The $\mathrm{P}$ values were calculated as follows: ns not significant; ${ }^{*} \mathrm{P}<0.05$; ${ }^{\star \star} \mathrm{P}<0.01$; $* \star \star \mathrm{P}$ $<0.001$. 